\title{
Importance of the physical exam and in-office tests in the evaluation of vulvovaginal irritation
}

\author{
Ashley Christensen, BSN, ${ }^{1}$ Michael Haugsdal, $\mathrm{MD},{ }^{2}$ and Noelle Bowdler, $\mathrm{MD}^{2}$
}

Keywords: Vulvovaginal irritation, physical exam, vaginosis, vaginitis, sexually transmitted infections

\begin{abstract} Background: Vulvovaginal irritation is a common gynecologic complaint. A number of factors may lead to a trial of therapy without undertaking a physical exam or diagnostic testing.

Case Report: A 45 year-old woman presented to our colposcopy clinic for evaluation of an abnormal Papanicolaou (Pap) test. She reported a one month history of vulvovaginal irritation, for which Premarin vaginal cream had been empirically prescribed. Examination of the external genitalia showed ulcers and erythema of the labia minora. Speculum exam was deferred because of the patient's discomfort. Wet mount microscopy from a vaginal swab revealed evidence of Trichomonas vaginalis, bacterial vaginosis (BV), and yeast. $A$ swab of the ulcers was sent for herpes simplex virus (HSV) polymerase chain reaction (PCR); this confirmed HSV-2. Treatment was initiated for each of these conditions, and the patient returned for colposcopy 21 days later.

Conclusion: This case illustrates the importance of the physical exam when evaluating a complaint of vulvovaginal irritation. In many cases, the cause(s) of vulvovaginal irritation can be identified based on physical exam findings and in-office testing with wet mount microscopy, vaginal $\mathrm{pH}$, and the amine "whiff" test. In some
\end{abstract}

cases, additional testing may be required to establish or confirm a diagnosis. Accurate diagnosis is essential not only to initiate appropriate therapy, but also to prevent the transmission of sexually transmitted infections. In some cases, this may decrease the delay in diagnosing vulvar gynecologic malignancies.

${ }^{1}$ University of lowa Carver College of Medicine,
lowa City, lowa
${ }^{2}$ University of lowa Hospitals and Clinics,
Department of Obstetrics and Gynecology, lowa
City, lowa

\section{Introduction}

Vulvovaginal irritation-burning, itching, or pain, abnormal vaginal discharge, and odor are common gynecologic complaints that lead women to seek care from their primary care provider or gynecologist. $^{1-4}$ A number of factors, including the availability of over-thecounter antifungal medications, ${ }^{5,6}$ and patient and provider time pressures and comfort with undertaking a pelvic exam, may lead to an empiric trial of therapy without undertaking a physical exam or

Please cite this paper as: Christensen A, Haugsdal ML, Bowdler NC. Importance of the physical exam and in-office tests in the evaluation of vulvovaginal irritation. Proc Obstet Gynecol. 2014;4(2): Article 11 [8 p.]. Available from: http://ir.uiowa.edu/pog/. Free full text article.

Corresponding author: Noelle C. Bowdler, MD, University of lowa Hospitals and Clinics, Department of Obstetrics and Gynecology, 200 Hawkins Drive, Iowa City, IA 52242. Telephone: 319-356-8353. noelle-bowdler@uiowa.edu.

Financial Disclosure: The authors report no conflict of interest.

Copyright: (c) 2014 Christensen et al. This is an open-access article distributed under the terms of the Creative Commons Attribution License, which permits unrestricted use, distribution, and reproduction in any medium, provided the original author and source are credited. 
diagnostic testing. While the most common causes of these symptoms are $\mathrm{BV}$, vaginal candidiasis and vaginal trichomoniasis, ${ }^{2,3}$ the differential diagnosis is broad, and includes vulvar infections, ${ }^{7}$ vulvovaginal atrophy, ${ }^{8,9}$ reactions to irritants or allergens, ${ }^{10,11}$ inflammatory conditions, ${ }^{12,13}$ premalignant, ${ }^{14}$ and malignant lesions.

\section{Case Description}

A 45 year-old G7P5 African American woman with hypertension, coronary artery disease, type 2 diabetes mellitus, morbid obesity, and anxiety presented to our colposcopy clinic for evaluation of high grade intraepithelial lesion (HSIL) on Pap test four months earlier. At presentation, she reported a history of significant discomfort due to vaginal irritation for approximately one month. Her primary care provider had empirically prescribed Premarin vaginal cream for this complaint.

Examination of the external genitalia showed several ulcers and areas of erythema on the labia minora. A speculum exam was deferred because of pain. A sample was collected from the vaginal introitus for wet mount, and a swab was collected from the vulvar ulcers for HSV PCR. Wet mount microscopy revealed trichomonads, clue cells, and branching pseudo-hyphae. The whiff test was positive and sample fluid had a $\mathrm{pH}$ of 5.5. In addition, HSV PCR returned positive for HSV-2. Gonorrhea and Chlamydia PCR were negative. The patient declined human immunodeficiency virus (HIV), hepatitis, and syphilis screening.

On review of records, it was noted that the patient had presented to her primary care provider 25 days prior to this visit with documented complaints of "vaginal itching, especially after bathing." A pelvic exam was not undertaken and the patient was given a prescription for Premarin vaginal cream, noting that she had a "gynecology appointment coming up."

The patient was counseled about the diagnoses of Trichomonas vaginalis, $\mathrm{BV}$, yeast, and HSV infection given the above findings. Oral metronidazole and fluconazole were prescribed. Furthermore, she was given valacyclovir for treatment of an initial HSV outbreak. She was asked to ensure that her partner undergo testing and treatment for trichomoniasis, in addition to abstaining from intercourse until both she and her partner were treated. She completed her medications as prescribed and was able to undergo colposcopy 21 days after her original visit.

\section{Discussion}

Vulvovaginal irritation—burning, itching, or pain, in addition to abnormal vaginal discharge and odor are common gynecologic complaints that lead women to seek care from their primary care physician or gynecologist. ${ }^{1-4}$ The underlying pathology in most cases involves BV, vaginal candidiasis (yeast), or trichomoniasis. ${ }^{2,3}$ Less often, herpes simplex virus $(\mathrm{HSV})^{7}$ and, in peri- or post-menopausal women, vulvovaginal atrophy $^{1,8,9}$ are the causes of vulvovaginal irritation. The differential diagnosis for these symptoms (Table 1) also includes conditions such as irritant or allergic contact dermatitis, ${ }^{10,11}$ lichen simplex chronicus, ${ }^{12,13}$ lichen sclerosus, ${ }^{12,13}$ lichen planus, ${ }^{12,13}$ vulvar 
intraepithelial neoplasia, ${ }^{14}$ and neoplasia. Despite sharing a common presentation, each of these vulvovaginal diseases is unique in its natural history, its effects on the local vaginal environment, the recommended medical management, and the potential sequela, making specific diagnosis essential.

\section{Table 1. Differential Diagnosis for Vulvovaginal Irritation}

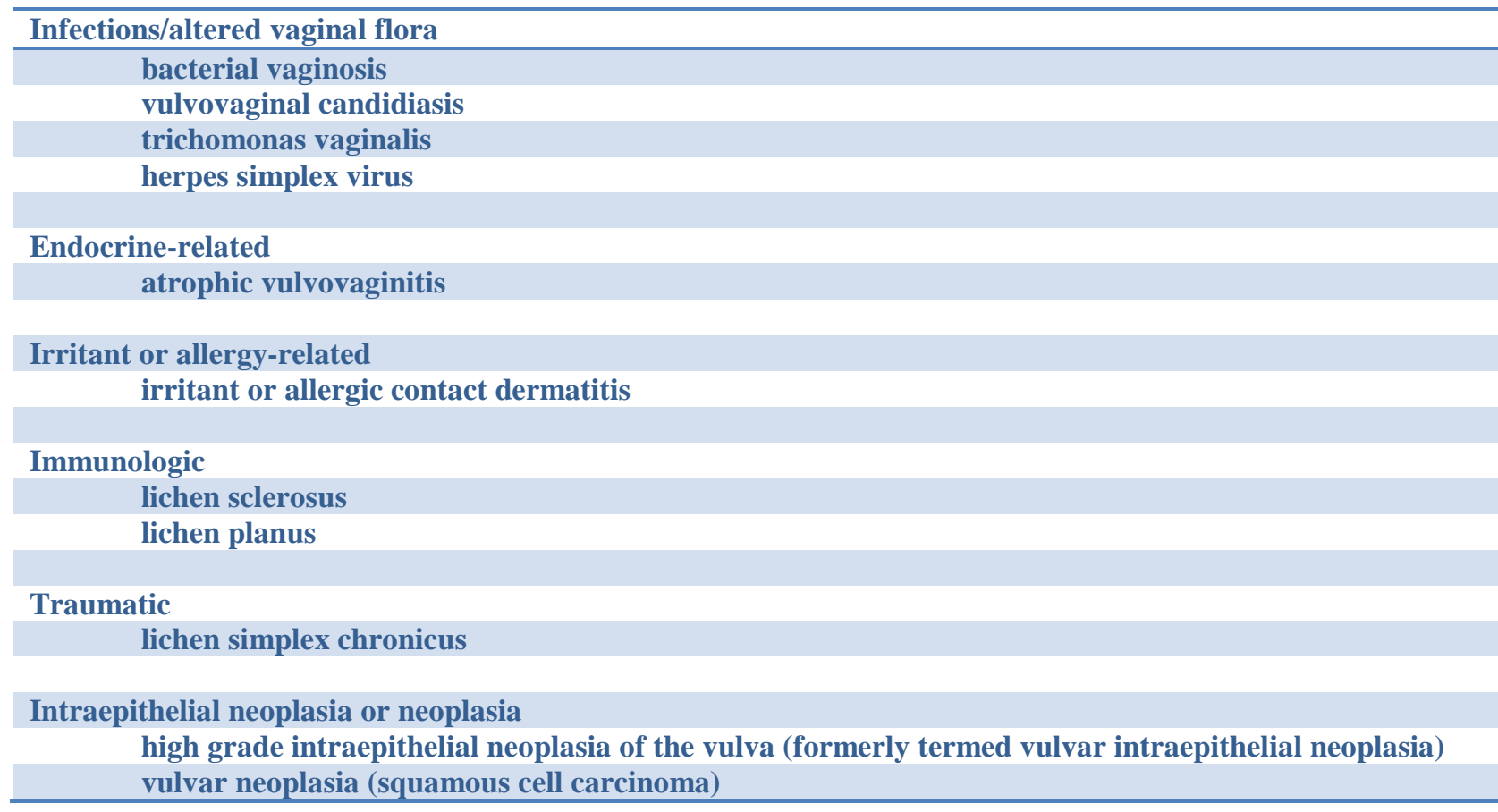

Unfortunately, the patient reported signs and symptoms are not specific enough to allow accurate diagnosis and appropriate management by the patient herself or in communication over the telephone with her healthcare provider. ${ }^{1,3-6}$ Hoffstetter et al. investigated telephone triage of patients with complaints suggestive of vaginitis. They reported the number of patients treated over the telephone has increased three-fold in recent years and that such limited assessment has led to an increase in misdiagnosis and ineffective treatment. Furthermore, they suggest that inappropriate treatment contributes to vulvar dermatitis and vulvodynia, in addition to selection for azole resistance and non-albicans species of yeast. ${ }^{4}$ Singh et al. compared agreement in the diagnosis of $\mathrm{BV}$, vaginal candidiasis, and Trichomonas vaginalis based on history by questionnaire and findings on a patientcollected vaginal swab with clinicianobtained history and findings on speculum and bimanual exams in patients with vaginal discharge. A diagnosis of BV was missed with history by questionnaire and patient-collected swabs in $32 \%$ of patients who were diagnosed with BV based on clinicianobtained history and physical exam. Diagnoses of vaginal candidiasis and 
Trichomonas vaginalis were missed with history by questionnaire and patientcollected swabs in $48 \%$ and $34 \%$ of clinician-diagnosed cases respectively. ${ }^{15}$ In addition to an accurate diagnosis of vaginosis or vaginitis, a pelvic exam in the setting of vulvovaginal irritation may result in a decreased delay in the diagnosis of gynecologic malignancy. ${ }^{16}$

This case illustrates that physical exam, in combination with simple in-office laboratory testing, will typically identify the cause(s) of vulvovaginal complaints. In some cases the physical exam findings will direct further testing such as HSV PCR or vulvar biopsy. Current literature supports this approach, strongly encouraging the clinician faced with vulvovaginal complaints to 'just take a look' before recommending medical management. ${ }^{1,2,15,17,18}$

\section{Evaluation of the patient with vulvovaginal irritation}

Problem-Focused History: A patient's self-reported signs and symptoms alone are not sufficient to correctly diagnose the cause(s) of vulvovaginal irritation, but they can contribute to the overall clinical picture. Vulvovaginal irritation may be characterized as itching, burning, or redness, and abnormal discharge may be described as thin, thick or cheesy (curd-like). Subjective report of an odor and the patient's perception of their underlying diagnosis may also be noteworthy. ${ }^{2} \mathrm{~A}$ history of exposure to new soaps, hygiene products, laundry products, vaginal douches, ${ }^{10}$ or toilet paper, ${ }^{11}$ use may be helpful. Finally, a history of recent antibiotic use, or of lesions noted visually or on palpation by the patient, can assist in the evaluation.
Physical Exam: A brief physical exam can confirm or clarify patient reported signs as well as provide an opportunity to make other observations to aid in the diagnosis. Examination of the vulva for erythema, swelling, atrophy, discharge in the region of the introitus, excoriation, and other discrete lesions is followed by visualization of the vaginal canal for evidence of atrophy, vaginal lesions, vaginal discharge, and cervical lesions and discharge. An odor may be appreciated Finally, a bimanual exam is performed to assess for tenderness. ${ }^{2,17}$

\section{In-Office (Point-of-care) Laboratory} Tests: During the physical exam a sample of vaginal fluid or discharge should be collected. This specimen is useful for analysis of vaginal $\mathrm{pH}$, amine whiff test with the addition of 10\% potassium hydroxide $(\mathrm{KOH})$, and microscopy to evaluate for clue cells, spores and pseudo-hyphae, and trichomonads. ${ }^{1,2}$ The number of white blood cells (WBC) per high-power field, the type of bacteria seen, and the maturity of the squamous epithelial cells also aid in the evaluation.

Several algorithms or clinical approaches are discussed in the literature, with the intent of judiciously arriving at an underlying etiology for vulvovaginal irritation., ${ }^{1,2}$ Each of the three most common diagnoses is characterized by a unique constellation of findings. The findings on physical exam and in-office testing often point to a diagnosis of one of the less common causes of vulvovaginal irritation as well, and direct further evaluation and treatment. 


\section{Physical exam and in-office laboratory findings with specific vulvovaginal conditions}

Bacterial Vaginosis: $\mathrm{BV}$ is the most common underlying etiology for patients with vulvovaginal complaints, affecting 40-50\% of women with these symptoms. Patients' reports of signs and symptoms are not typically useful indicators of BV, with the exception of a characteristic "fishy" vaginal odor that is highly associated with $\mathrm{BV} .{ }^{2,17,18}$ Examination often reveals a thin white discharge that may be visible at the introitus. Vaginal $\mathrm{pH}$ of $>4.5$ is inherent to $\mathrm{BV}$, but this finding also overlaps with trichomoniasis. ${ }^{1,2}$ A positive whiff test is associated with BV, as are microscopy findings of clue cells and decreased lactobacillii. ${ }^{2} \mathrm{~A}$ variety of bacterial forms is typically seen in the background including cocci, coccobacilli, and curved rods. A diagnosis of BV may be established based on the presence of at least two of four criteria (Amsel criteria): thin white vaginal discharge, $\mathrm{pH}>4.5$, positive whiff test, and the presence of clue cells on wet prep microscopy. ${ }^{17}$

Vaginal Candidiasis: Patient reported signs and symptoms may be the most helpful in diagnosing vaginal candidiasis, but should not be the sole source of clinical reasoning. Vaginal itching is highly sensitive for candidiasis as $70-90 \%$ of patients with yeast infection report itching explicitly and a lack thereof decreases the likelihood of this diagnosis. ${ }^{2}$ The absence of an odor noted by the patient or clinician and selfdiagnosis of 'another yeast infection' in a patient with a history of previously correctly diagnosed vaginal candidiasis both increase the likelihood of the diagnosis as well. ${ }^{1,5,6}$ The finding of a thick, curd-like discharge as well as erythema and excoriation (secondary to itching) are associated with candidiasis. ${ }^{2}$ Vaginal $\mathrm{pH}$ is typically normal (3.5-4.5) and, while pseudo-hyphae and/or spores on microscopy are diagnostic, their absence does not exclude the diagnosis. ${ }^{1,2}$ A vaginal yeast culture is helpful when pseudo-hyphae or spores are not seen on microscopy. ${ }^{17,18}$

Vaginal Trichomoniasis: As with BV, patient reported signs and symptoms are not specific for trichomoniasis and the vaginal $\mathrm{pH}$ is typically $>4.5$ with both of these conditions. While an increase in the number of leukocytes relative to epithelial cells is typical, the finding of motile trichomonads is the only specific diagnostic finding on microscopy. $2,17,18$ As the sensitivity of microscopy for diagnosing trichomoniasis varies from 50 to $80 \%$, rapid antigen testing, a nucleic acid probe, or PCR can be used to aid in the diagnosis of this condition. ${ }^{17,18}$

Herpes Simplex Virus (HSV): Vulvar lesions that are characteristic of HSV include small vesicles that typically occur in clusters, and shallow ulcerations that may have an overlying exudate. Particularly with a primary episode, there may be surrounding erythema or edema. While there are no accurate in-office tests to establish the diagnosis of HSV, PCR from a swab of fluid from a vesicle or ulcer can be used to rapidly confirm a clinical suspicion of $\mathrm{HSV}^{7}$

Atrophic Vaginitis: Vulvar atrophy is characterized by thinning of the epithelium of the labia minora, and vaginal atrophy is associated with thinning of the vaginal epithelium. On 
examination, the vaginal mucosa appears red or pale and smooth rather than rugated. The vagina may appear dry or have evidence of a thin yellow discharge. Microscopy from a vaginal swab typically shows parabasal and intermediate squamous cells in combination with, or instead of, mature squamous cells. The vaginal $\mathrm{pH}$ is typically $>4,5$. $^{8,9}$

Contact Dermatitis: Irritant or allergic contact dermatitis is characterized by erythema and edema of the labia minora and an absence of abnormal findings on vaginal microscopy and vaginal yeast culture. A biopsy may help to establish the diagnosis. Skin testing can assist in identifying causative allergens. ${ }^{10,11}$

Vestibulodynia: Examination of the patient with vestibulodynia shows a normal exam with the exception of possible erythema surrounding the vestibular glands in the region of the introitus. These areas are typically tender when a cotton-tipped applicator is applied to the sites. Again, vaginal microscopy shows normal findings and vaginal yeast culture is typically negative.

Lichen sclerosus: Examination of the patient with lichen sclerosus shows a silver or white appearance to the vulva that is shiny or may appear "wrinkled", with an appearance similar to that of tissue paper; erythema is often present. The labia minora, clitoris, and posterior fourchette are most commonly affected, but changes may be seen in the perianal area as well. Biopsy is required to establish the diagnosis. ${ }^{12,13}$

Lichen planus: Vulvovaginal lichen planus is characterized most often by introital erosions. There may be white reticular lesions. In addition, there may be vaginal erosions, adhesions, and a vaginal discharge, the microscopy of which shows large numbers of white blood cells and parabasal squamous epithelial cells. Papulosquamous and hypertrophic forms also occur. A vulvar biopsy is generally required to establish the diagnosis. ${ }^{12,13}$

Lichen simplex chronicus: Lichen simplex chronicus is caused by scratching the vulva and is characterized by thickening of the skin (lichenification) with increased skin markings and excoriations. ${ }^{12,13}$ Wet mount microscopy or yeast culture may reveal yeast as the cause of the scratching, ${ }^{13}$ or an irritant or allergen may be identified based on the patient's history or skin testing.

High Grade Intraepithelial Neoplasia of the Vulva (formerly termed Vulvar Intraepithelial Neoplasia (VIN)) and Invasive Carcinoma: With high grade intraepithelial neoplasia of the vulva, raised or papillary white or pigmented lesions may be evident. These may be single or multi-focal. Application of dilute (3\%) acetic acid may result in a more opaque (whiter) appearance to the lesions, although this is not a specific finding on the vulva. ${ }^{14}$ The diagnosis is established with biopsy. Raised white, red, or pigmented lesions, and ulcerated lesions are characteristic of invasive vulvar carcinoma. Again, the diagnosis is established with biopsy.

\section{Significance of the present case}

In the case described here, the patient had lesions that were suggestive of HSV on exam as well as vulvar erythema. 
Microscopy of the patient's vaginal discharge showed evidence of trichomonads, clue cells, and pseudohyphae. PCR from the vulvar lesions confirmed a diagnosis of HSV. Treatment was initiated based on the physical exam and wet mount microscopy findings, with the addition of valacyclovir when the HSV PCR result returned later the same day. The patient's symptoms improved, such that she was able to undergo colposcopy a short time later. It was recommended that the patient's partner undergo evaluation and treatment for trichomoniasis. The diagnosis of HSV resulted in counselling about ways to decrease the likelihood of transmission to others.

As demonstrated by the presented case, a diagnosis that is based on symptoms, in the absence of a physical exam, may be inaccurate and can lead to treatment that is inappropriate and even detrimental to personal and public health. ${ }^{4}$

\section{Conclusion}

In most cases, the cause(s) of vulvovaginal irritation can be rapidly and accurately diagnosed using a combination of pelvic exam and in-office testing. In some cases, additional testing with a yeast culture, HSV PCR, or biopsy may be helpful. While there are barriers to the early evaluation of vulvovaginal irritation by a clinician, this will lead to cost savings related to the inappropriate use of over-the-counter products and help to avoid delays in the diagnosis of transmissible conditions or potentially serious conditions such as vulvar neoplasia.
Acknowledgments: We would like to acknowledge the contributions of Timothy Bahr, $B S$ and Erica Hofland, MD to the concept of and initial work on this case report.

\section{References}

1. Nyirjesy P, Sobel JD. Advances in diagnosing vaginitis: development of a new algorithm. Curr Infect Dis Rep. 2005 Nov;7(6):458-62. http://dx.doi.org/10.1007/s11908-0050048-3. PubMed PMID: 16225784.

2. Anderson $M R$, Klink $K$, Cohrssen $A$. Evaluation of vaginal complaints. JAMA. 2004 Mar 17;291(11):1368-79. http://dx.doi.org/10.1001/jama.291.11.13 68. PubMed PMID: 15026404.

3. Theroux R. Women's self-diagnostic skills: developing the science. Nurs Womens Health. 2010 Oct;14(5):399$404 . \quad$ doi: 10.1111/j.1751486X.2010.01579.x. PubMed PMID: 20955529.

4. Hoffstetter S, Barr S, LeFevre C, Gavard JA. Telephone triage: diagnosis of candidiasis based upon self-reported vulvovaginal symptoms. J Low Genit Tract Dis. 2012 Jul;16(3):251-5. doi: 10.1097/LGT.0b013e31823f9068.

PubMed PMID: 22453756.

5. Ferris DG, Nyirjesy P, Sobel JD, Soper D, Pavletic A, Litaker MS. Over-thecounter antifungal drug misuse associated with patient-diagnosed vulvovaginal candidiasis. Obstet Gynecol. $2002 \quad$ Mar;99(3):419-25. http://dx.doi.org/10.1016/S0029-

7844(01)01759-8. PubMed PMID: 11864668.

6. McCaig LF, McNeil MM. Trends in prescribing for vulvovaginal candidiasis in the United States. Pharmacoepidemiol Drug Saf. 2005 Feb;14(2):113-20. http://dx.doi.org/10.1002/pds.960. PubMed PMID: 15386715. 
7. Garland SM, Steben M. Genital herpes. Best Pract Res Clin Obstet Gynaecol. 2014 Aug 4. pii: S1521-6934(14)001394. doi: 10.1016/j.bpobgyn.2014.07.015. [Epub ahead of print] PubMed PMID: 25153069.

8. Mac Bride MB, Rhodes DJ, Shuster LT. Vulvovaginal atrophy. Mayo Clin Proc. 2010 Jan;85(1):87-94. doi: 10.4065/mcp.2009.0413. Review. PubMed PMID: 20042564.

9. Weber MA, Limpens J, Roovers JP. Assessment of vaginal atrophy: a review. Int Urogynecol J. 2014 Jul 22. [Epub ahead of print] DOI10.1007/s00192-014-2464-0.

PubMed PMID: 25047897.

10. Bauer A, Rodiger C, Greif C, Kaatz M, Elsner P. Vulvar dermatoses-irritant and allergic contact dermatitis of the vulva. Dermatology. 2005;210(2):143-9. http://dx.doi.org/10.1159/000082570.

PubMed PMID: 15724097.

11. Majerovich JA, Canty A, Miedema B. Chronic vulvar irritation: could toilet paper be the culprit? Can Fam Physician. 2010 Apr;56(4):350-2. PubMed PMID: 20393094.

12. Moyal-Barracco $M$, Wendling J. Vulvar dermatosis. Best Pract Res Clin Obstet Gynaecol. 2014 Jul 18. pii: S15216934(14)00129-1.

doi: 10.1016/j.bpobgyn.2014.07.005. [Epub ahead of print] PubMed PMID: 25132276.

13. Thorstensen KA, Birenbaum DL. Recognition and management of vulvar dermatologic conditions: lichen sclerosus, lichen planus, and lichen simplex chronicus. J Midwifery Womens Health. 2012 May-Jun;57(3):260-75. doi: 10.1111/j.1542-2011.2012.00175.x.

PubMed PMID: 22594865.
14. Preti M, Scurry J, Marchitelli CE, Micheletti L. Vulvar intraepithelial neoplasia. Best Pract Res Clin Obstet Gynaecol. 2014 Jul 18. pii: S15216934(14)00134-5. doi: 10.1016/j.bpobgyn.2014.07.010. [Epub ahead of print] PubMed PMID: 25106700 .

15. Singh RH, Zenilman JM, Brown KM, Madden T, Gaydos C, Ghanem KG. The role of physical examination in diagnosing common causes of vaginitis: a prospective study. Sex Transm Infect. 2013 May;89(3):185-90. doi: 10.1136/sextrans-2012-050550. Epub 2012 Sep 27. PubMed PMID: 23019659.

16. Vandborg MP, Christensen RD, Kragstrup J, Edwards K, Vedsted $\mathrm{P}$, Hansen DG, Mogensen O. Reasons for diagnostic delay in gynecological malignancies. Int J Gynecol Cancer. 2011 Aug;21(6):967-74. doi: 10.1097/IGC.0b013e31821d2770. PubMed PMID: 21792008.

17. Hainer BL, Gibson MV. Vaginitis: diagnosis and treatment. Am Fam Physician. 2011 Apr 1;83(7):807-15. PubMed PMID: 21524046.

18. Mashburn J. Vaginal infections update. J Midwifery Womens Health. 2012 NovDec;57(6):629-34. doi: 10.1111/j.15422011.2012.00246.x. Epub 2012 Oct 24. PubMed PMID: 23094602. 